\title{
FINDING OCTONION ALGEBRAS IN ASSOCIATIVE ALGEBRAS
}

\author{
JOHN R. FAULKNER
}

(Communicated by Donald S. Passman)

\begin{abstract}
An embedding of octonion algebras in central simple degree three associative algebras is given.
\end{abstract}

Using standard operations on trace 0 elements of a simple 9-dimensional associative algebra (such as $3 \times 3$ matrices) over a field of characteristic not 3 containing a primitive cube root of 1 , we obtain in the Theorem the somewhat surprising embedding of an octonion algebra in an associative algebra. One clue to this embedding is that the Tits construction in [4] applied to $F \oplus \mathscr{O}$ ( $\mathcal{O}$ split octonion) or to $F_{3}(3 \times 3$ matrices $)$ gives the same 27-dimensional exceptional Jordan algebra, suggesting that $F \oplus \mathscr{O}$ is related to $F_{3}$. A similar embedding of the 27-dimensional exceptional Jordan algebra $\mathcal{J}$ as trace 0 elements in the degree 4 special Jordan algebra $\mathscr{H}_{4}$ of $4 \times 4$ hermitian quaternionic matrices was previously known (see [ 1 and 2]). In this case, a relation between $F \oplus \mathscr{J}$ and $\mathscr{H}_{4}$ was suggested by the Cayley-Dickson process in [1] which applied to $F \oplus \mathscr{J}$ or to $\mathscr{H}_{4}$ gives the same 56-dimensional structurable algebra.

Our approach is to show that the set $A_{0}$ of trace 0 elements of a separable degree 3 alternative algebra $A$ carries a nondegenerate quadratic form $S$ permitting composition $S(x * y)=S(x) S(y)$, from which it follows that an isotope of $\left(A_{0},{ }^{*}\right)$ is a unital composition algebra. If $A$ has dimension 9 , then $A_{0}$ has dimension 8, and the composition algebra must be octonion.

We begin by establishing some basic facts about degree 3 alternative algebras.

LEMMA. Let $A$ be a finite-dimensional alternative algebra of degree 3, with generic minimum polynomial $p_{x}(\lambda)=\lambda^{3}-T(x) \lambda^{2}+S(x) \lambda-N(x) 1$. Setting $x^{\#}=$ $x^{2}-T(x) x+S(x) 1, S(x, y)=S(x+y)-S(x)-S(y)$, and $x \times y=(x+y)^{\#}-x^{\#}-y^{\#}$, we have

(a) $N(x y)=N(x) N(y)$,

(b) $S(1)=T(1)=3, N(1)=1$,

(c) $S(x)=T\left(x^{\#}\right)$

(d) $S(x, y)=T(x \times y)$,

(e) $S(x, 1)=2 T(x)$,

(f) $T(x) T(y)=T(x y)+T(x \times y)$,

(g) $2 S(x)=T(x)^{2}-T\left(x^{2}\right)$,

(h) $T(x y)=T(y x)$,

(i) $x \times y=x y+y x-T(x) y-T(y) x+(T(x) T(y)-T(x y)) 1$,

(j) $x^{\# \#}=N(x) x$,

Received by the editors March 15, 1988.

1980 Mathematics Subject Classification (1985 Revision). Primary 17A75; Secondary 17C40. 
(k) $(x \times y)^{\#}+x^{\#} \times y^{\#}=T\left(x^{\#} y\right) y+T\left(x y^{\#}\right) x$,

(l) $(x y)^{\#}=y^{\#} x^{\#}$,

(m) $S(x y)=S(y x)$.

PROOF. We may assume the field is infinite and use differential calculus as in [5] where (a), (b) and $p_{x}(\lambda)=N(\lambda 1-x)$ are proved. We have $x^{-1}=N(x)^{-1} x^{\#}$ if $N(x) \neq 0$, since $x x^{\#}-N(x) 1=x^{\#} x-N(x) 1=p_{x}(x)=0$. Since $N(x+\lambda y)=$ $\lambda^{3} N(y)+\left.\lambda^{2} \partial_{x} N\right|_{y}+\left.\lambda \partial_{y} N\right|_{x}+N(x)$, we see $T(x)=\left.\partial_{x} N\right|_{1}$ and $S(x)=\left.\partial_{1} N\right|_{x}$. Since $N(x+\lambda y)=N\left(x y^{-1}+\lambda 1\right) N(y)$, we also see $\left.\partial_{x} N\right|_{y}=T\left(x y^{-1}\right) N(y)=T\left(x y^{\#}\right)$, if $N(x) \neq 0$. By density, $\left.\partial_{x} N\right|_{y}=T\left(x y^{\#}\right)$. Now $x=1$ gives (c), and (d) follows by linearization. We see $x \times y=\left.\partial_{x} \#\right|_{y}$ so $T(x(y \times z))=\left.\partial_{y} \partial_{x} N\right|_{z}$ is symmetric in $x, y$, and $z$. In particular, $S(x, 1)=\left.\partial_{x} S\right|_{1}=\left.\partial_{x} \partial_{1} N\right|_{1}=T(x(1 \times 1))=2 T(x)$ showing (e), since $1 \times 1=2\left(1^{\#}\right)=2$ by the definition of $X$, \#, and (b). We also compute $x \times 1=\left.\partial_{x} \#\right|_{1}=x 1+1 x-T(x) 1-T(1) x+S(x, 1) 1=T(x) 1-x$. Hence, $T(x \times y)=T((x \times 1) y)=T(T(x) y-x y)=T(x) T(y)-T(x y)$ showing (f). Clearly, (g) and (h) follow from (f). Moreover, (i) follows from (d) and (f). Since $x^{\# \#}=\left(N(x) x^{-1}\right)^{\#}=N(x)^{2} N\left(x^{-1}\right)\left(x^{-1}\right)^{-1}$ and $N(x) N\left(x^{-1}\right)=N(1)=1$, we see $(\mathrm{j})$ holds by density. Moreover, $(\mathrm{k})$ is the coefficient of $\lambda^{2}$ in $(\mathrm{j})$ with $x$ replaced by $x+\lambda y$, using $\left.\partial_{x} N\right|_{y}=T\left(x y^{\#}\right)$. To get $(\mathrm{l})$, note $(x y)^{\#}=N(x y)(x y)^{-1}=$ $N(x) N(y) y^{-1} x^{-1}=y^{\#} x^{\#}$ and use density. Finally, (m) follows from (c), (l), and (h).

Before looking at the general situation, we motivate the definition of $x * y$ in the following Theorem by investigating the relationship between $A_{0}$ and $\mathscr{O}$ in the particular degree 3 alternative algebra $A=F \oplus \mathscr{O}$. If $x=(\alpha, a)$, then $T(x)=$ $\alpha+t(a), S(x)=\alpha t(a)+n(a)$, and $N(x)=\alpha n(a)$, in terms of the trace $t$ and the norm $n$ on $\mathscr{O}$. The projection $x \rightarrow a$ restricted to $A_{0}$ is an isometry of $\left(A_{0}, S\right)$ with $\left(\mathscr{O}, n-t^{2}\right)$ since $\alpha=-t(a)$ on $A_{0}$. Also, if $\omega+\omega^{2}+1=0$ in $F$, then $a \rightarrow a+\omega t(a)$ is an isometry of $\left(\mathscr{O}, n-t^{2}\right)$ with $(\mathscr{O}, n)$. Combining, we get an isometry $\theta:(-t(a), a) \rightarrow a+\omega t(a)$ of $\left(A_{0}, S\right)$ with $(\mathscr{O}, n)$. Since $n$ admits composition relative to the product in $\mathscr{O}, S$ automatically admits composition $S(x * y)=S(x) S(y)$ relative to the product

$$
x * y=\theta_{1}^{-1}\left(\theta_{2}(x) \theta_{3}(y)\right)
$$

which has been pulled back from $\mathscr{O}$ by any isometries $\theta_{i}$ of $\left(A_{0}, S\right)$ with $(\mathscr{O}, n)$. In particular, taking all $\theta_{i}=\theta$ above gives a product $x \square y$ on $A_{0}$ isomorphic to that on $\mathscr{O}$. We would like to express $x \square y$ in terms of the original product on $A$. For $y=(\beta, b) \in A_{0}$, a direct computation (using $a+\bar{a}=t(a)$ ) shows

$$
\theta(x) \theta(y)=-\omega^{2} a b+\omega b a-\gamma
$$

where $\gamma=\omega t(a b)+t(a) t(b)$. On the other hand, if $x \rightarrow x_{0}$ is the projection onto $A_{0}$, i.e. $x_{0}=x-\frac{1}{3} T(x) 1$, then we also have

$$
\theta\left((x y)_{0}\right)=-\bar{b} \bar{a}+\rho \gamma,
$$

where $\rho=\left(\omega-\omega^{2}\right)^{-1}$. Consequently, $\theta\left(\left(\omega x y-\omega^{2} y x\right)_{0}\right)=-\omega \bar{b} \bar{a}+\omega^{2} \bar{a} \bar{b}+\gamma=$ $(j \theta(x))(-j \theta(y))$ where $j(a)=\bar{a}$. Taking $\theta_{1}=\theta, \theta_{2}=j \theta$, and $\theta_{3}=-j \theta$ in (1), shows $S$ admits composition relative to $x * y=\left(\omega x y-\omega^{2} y x\right)_{0}$. Moreover, if $u \in A_{0}$ with $\theta(u)=1$, then $\theta(x * u)=-j \theta(x)=\theta(u * x)$ so $\theta((x * u) *((-u) * y))=\theta(x) \theta(y)$ and $x \square y$ is the product in the $(u,-u)$-isotope of $\left(A_{0}, *\right)$. This suggests the product * used in the Theorem. 
THEOREM. If $A$ is a finite-dimensional alternative algebra of degree $\$$ over a field $F$ of characteristic not 3 containing an element $\omega$ with $\omega^{2}+\omega+1=0$, then the coefficient $S(x)$ of $\lambda$ in the generic minimum polynomial permits composition $S(a * b)=S(a) S(b)$ on $A_{0}=\{x: T(x)=0\}$ relative to the product $a * b=(\omega a b-$ $\left.\omega^{2} b a\right)_{0}$ where $x_{0}=x-\frac{1}{3} T(x) 1$. If $A$ is separable over $F$, then the quadratic form $S$ is nondegenerate, and $\left(A_{0}, *\right)$ is a quasi-composition algebra with a unital isotope which is a composition algebra. If $A$ also has dimension 9 , then the isotope is an octonion algebra.

$$
\begin{aligned}
& \text { ProOF. We have } S(a) S(b)=T\left(a^{\#}\right) T\left(b^{\#}\right) \quad(\text { by }(\mathrm{c})) \\
& =T\left(a^{\#} b^{\#}\right)+T\left(a^{\#} \times b^{\#}\right) \quad(\text { by }(\mathrm{f})) \\
& \left.=T\left(a^{\#} b^{\#}\right)-T\left((a \times b)^{\#}\right)\right) \\
& \text { (by } T \text { applied to (k) using } a, b \in A_{0} \text { ) } \\
& =S(a b)-S(a \times b) \quad \text { by }(\mathrm{c}),(\mathrm{l}), \text { and }(\mathrm{m})) \\
& =S(a b)-S(a b+b a-T(a b) 1) \quad(\text { by }(\mathrm{i})) \\
& =S(a b)-S(a b)-S(b a)-S(a b, b a)+T(a b) S(a b+b a, 1) \\
& -T(a b)^{2} S(1) \\
& =-S(a b)-S(a b, b a)+T(a b)^{2} \\
& \text { (by (b), (m), (e), and (h)). }
\end{aligned}
$$

On the other hand, $S\left(x_{0}\right)=S\left(x-\frac{1}{3} T(x) 1\right)=S(x)-\frac{1}{3} S(x, 1) T(x)+\frac{1}{9} T(x)^{2} S(1)=$ $S(x)-\frac{1}{3} T(x)^{2}$ by (e) and (b). Thus, $S(a * b)=S\left(\omega a b-\omega^{2} b a\right)-\frac{1}{3} T\left(\omega a b-\omega^{2} b a\right)^{2}=$ $\omega^{2} S(a b)+\omega^{4} S(b a)-\omega \omega^{2} S(a b, b a)-\frac{1}{3}\left(\omega-\omega^{2}\right)^{2} T(a b)^{2}($ by $(\mathrm{h}))=-S(a b)-S(a b, b a)+$ $T(a b)^{2}$, since $\omega^{3}=1, \omega+\omega^{2}=-1$, and $\left(\omega-\omega^{2}\right)^{2}=-3$. If $A$ is separable over $F$, then $T(x y)$ is nondegenerate on $A$ and on $A_{0}$. Since $S(x, y)=-T(x y)$ on $A_{0}$ by (d) and (f), $S$ is also nondegenerate on $A_{0}$. The remainder now follows from Kaplansky [3]. Indeed, whenever $S(u) \neq 0$, we get a unital composition algebra from the isotope with product $a \square b=\left(R_{u}^{*-1} a\right) *\left(L_{u}^{*-1} b\right)$ with unit $u * u$. Here $R_{u}^{*}$ and $L_{u}^{*}$ denote the right and left multiplication in $\left(A_{0}, *\right)$.

EXAMPLE. If $A=F_{3}$ and $u=\operatorname{diag}\{2 \rho,-\rho,-\rho\}$ for $\rho=\left(\omega-\omega^{2}\right)^{-1}$, then $\left(F_{3}\right)_{0}$ is a split octonion algebra with identity element $u$ and norm form $\operatorname{tr}(\operatorname{adj} a)$ relative to the product $a \square b=(u * a) *(b * u)$ where $x * y=\omega x y-\omega^{2} y x+\rho \operatorname{tr}(x y)$ Id.

ProOF. We use $A=F_{3}$ in the Theorem, so $T(x)=\operatorname{tr}(x), N(x)=\operatorname{det}(x), S(x)=$ $\operatorname{tr}(\operatorname{adj} x)$, and $x^{\#}=\operatorname{adj} x$, the matrix adjoint. Since $\rho^{-2}=-3$, we see $-\frac{1}{3}\left(\omega-\omega^{2}\right)=\rho^{2} \rho^{-1}=\rho$ and $x * y=\left(\omega x y-\omega^{2} y x\right)_{0}=\omega x y-\omega^{2} y x+\rho \operatorname{tr}(x y)$ Id. Let $e_{1}=\operatorname{diag}\left\{1, \omega, \omega^{2}\right\}, e_{2}=\operatorname{diag}\left\{1, \omega^{2}, \omega\right\}$, so $e_{i} \in A_{0}$ have $e_{i} e_{j}=\operatorname{Id}, N\left(e_{i}\right)=1$, $e_{i}^{\#}=e_{i}^{2}=e_{j}$ for $i \neq j$. We see $e_{i} * e_{i}=\left(\omega-\omega^{2}\right)\left(e_{i}^{2}\right)_{0}=\rho^{-1} e_{j}, i \neq j$, and $e_{i} * e_{j}=\left(\omega-\omega^{2}\right)\left(e_{i} e_{j}\right)_{0}=0$. If $u=\rho e_{1}+\rho e_{2}=\operatorname{diag}\{2 \rho,-\rho,-\rho\}$, we see $u * e_{i}=e_{i} * u=e_{j}$ and $u * u=u$. If $L_{x}^{*} y=R_{y}^{*} x=x * y$ on $A_{0}$, we note that $L_{u}^{*}$ is the inverse of $R_{u}^{*}$ on $F e_{1}+F e_{2}$. In general, if $L_{x} y=R_{y} x=x y$, and if $d=\operatorname{diag}\left\{\lambda_{1}, \lambda_{2}, \lambda_{3}\right\}$, then $L_{d}$ and $R_{d}$ act on matrices $F E_{i j}(i \neq j)$ by multiplication by $\lambda_{i}$ and $\lambda_{j}$. On $F E_{i j}$, we have $L_{d}^{*}=\omega L_{d}-\omega^{2} R_{d}=\left(\omega \lambda_{i}-\omega^{2} \lambda_{j}\right)$ Id and $R_{d}^{*}=-\omega^{2} L_{d}+\omega R_{d}=\left(-\omega^{2} \lambda_{i}+\omega \lambda_{j}\right) \mathrm{Id}$, so $L_{d}^{*} R_{d}^{*}=-\left(\lambda_{i}^{2}+\lambda_{j}^{2}+\lambda_{i} \lambda_{j}\right)$ Id. Note that for $d=u, \lambda_{1}=2 \rho, \lambda_{2}=-\rho, \lambda_{3}=-\rho$, this is $-\left((2 \rho)^{2}+(-\rho)^{2}+\left(-2 \rho^{2}\right)\right)$ or $-\left((-\rho)^{2}+(-\rho)^{2}+(\rho)^{2}\right)$ both of which are $-3 \rho^{2}=1$. Thus, $R_{u}^{*}=\left(L_{u}^{*}\right)^{-1}$. 
Since $S(u)=\operatorname{tr}(\operatorname{adj}(u))=\operatorname{tr} \operatorname{diag}\left\{\rho^{2},-2 \rho^{2},-2 \rho^{2}\right\}=1$, we get $\left(F_{3}\right)_{0}$ with product $a \square b=\left(R_{u}^{*-1} a\right) *\left(L_{u}^{*-1} b\right)=(u * a) *(b * u)$ is an eight-dimensional unital composition algebra from [3]. Since $S\left(e_{1}\right)=\operatorname{tr}\left(\operatorname{adj}\left(e_{1}\right)\right)=\operatorname{tr}\left(e_{2}\right)=0$, it is a split octonion algebra.

I wish to thank Kevin McCrimmon for suggestions improving the exposition of this paper.

\section{REFERENCES}

1. B. N. Allison and J. R. Faulkner, A Cayley-Dickson process for a class of structurable algebras, Trans. Amer. Math. Soc. 283 (1984), 185-210.

2. J. R. Faulkner, A geometric construction of Moufang planes (To appear in Geometrice Dedicata).

3. I. Kaplansky, Infinite-dimensional quadratic forms admitting composition, Proc. Amer. Math. Soc. 4 (1953), 956-960.

4. K. McCrimmon, The Freudenthal-Springer-Tits constructions of exceptional Jordan algebras, Trans. Amer. Math. Soc. 139 (1969), 495-510.

5. __, Generically algebraic algebras, Trans. Amer. Math. Soc. 127 (1967), 527-551.

Department of Mathematics, University of Virginia, Charlottesville, VirGINIA 22903 